\title{
Identification of significant gene and pathways involved in HBV-related hepatocellular carcinoma by bioinformatics analysis
}

\author{
Shucai Xie ${ }^{1}$, Xili Jiang ${ }^{2}$, Jianquan Zhang ${ }^{1}$, Shaowei Xie ${ }^{1}$, Yongyong Hua ${ }^{1}$, Rui Wang ${ }^{1}$, Yijun Yang ${ }^{\text {Corresp. } 1}$ \\ ${ }^{1}$ Department of Hepatobiliary Surgery, Haikou People's Hospital /Affiliated Haikou Hospital of Xiangya Medical College, Central South University, Haikou, \\ Hainan, China \\ 2 Department of Radiology, The Second People's Hospital of Hunan Province/Brain Hospital of Hunan Province, Changsha, China \\ Corresponding Author: Yijun Yang \\ Email address: dryyj@163.com
}

Background: Hepatocellular carcinoma $(\mathrm{HCC})$ is a common malignant tumor affecting the digestive system and causes serious financial burden worldwide. Hepatitis B virus (HBV) is the main causative agent of HCC in China. The present study aimed to investigate the potential mechanisms underlying HBV-related HCC and to identify core biomarkers by integrated bioinformatics analyses. Methods: In the present study, HBV-related HCC GSE19665, GSE55092, GSE94660 and GSE121248 expression profiles were downloaded from the Gene Expression Omnibus database. These databases contain data for 299 samples, including 145 HBV-related HCC tissues and 154 non-cancerous tissues (from patients with chronic hepatitis B). The differentially expressed genes (DEGs) from each database were integrated and analyzed using the RobustRankAggreg (RRA) method and $R$ software, and the integrated DEGs were identified. Subsequently, the gene ontology (GO) functional annotation and Kyoto Encyclopedia of Genes and Genomes (KEGG) pathway analysis were performed using the DAVID online tool, and the protein-protein interaction (PPI) network was constructed using STRING and visualized using Cytoscape software. Finally, hub genes were identified, and the cBioPortal online platform was used to analyze the association between the expression of hub genes and prognosis in HBV-related HCC. Results: First, 341 DEGs (117 upregulated and 224 downregulated) were identified from the four datasets. Next, GO analysis showed that the upregulated genes were mainly involved in cell cycle, mitotic spindle, and adenosine triphosphate binding. The majority of the downregulated genes were involved in oxidation reduction, extracellular region, and electron carrier activity. Signaling pathway analysis showed that the integrated DEGs shared common pathways in retinol metabolism, drug metabolism, tryptophan metabolism, caffeine metabolism, and metabolism of xenobiotics by cytochrome P450. The integrated DEG PPI network complex comprised 288 nodes, and two important modules 
with high degree were detected using the MCODE plug-in. The top ten hub genes identified from the PPI network were SHCBP1, FOXM1, KIF4A, ANLN, KIF15, KIF18A, FANCI, NEK2, ECT2, and RAD51AP1. Finally, survival analysis revealed that patients with HBV-related HCC showing altered ANLN and KIF18A expression profiles showed worse disease-free survival. Nonetheless, patients with FOXM1, NEK2, RAD51AP1, ANLN, and KIF18A alterations showed worse overall survival. Conclusions: The present study identified key genes and pathways involved in HBV-related HCC, which improved our understanding of the mechanisms underlying the development and recurrence of HCC and identified candidate targets for the diagnosis and treatment of HBV-related HCC. 


\section{Identification of significant gene and pathways involved in HBV-related hepatocellular carcinoma by bioinformatics analysis}

Shucai Xie ${ }^{1}$, Xili Jiang ${ }^{2}$, Jianquan Zhang ${ }^{1}$, Shaowei Xie ${ }^{1}$, Yongyong Hua ${ }^{1}$, Rui Wang ${ }^{1}$, Yijun Yang*1

1Department of Hepatobiliary Surgery, Haikou People's Hospital /Affiliated Haikou

Hospital of Xiangya Medical College, Central South University, Haikou, Hainan 570208, P.R. China.

2 Department of Radiology, The Second People's Hospital of Hunan Province/Brain Hospital of Hunan Province, Changsha, Hunan 410007, P.R. China.

*Corresponding Author:

Yijun Yang

56 Renmin Avenue, Haikou, Hainan, 570208, P.R. China.

E-mail address: dryyj@163.com.

\section{Abstract}

Background: Hepatocellular carcinoma (HCC) is a common malignant tumor affecting the digestive system and causes serious financial burden worldwide. Hepatitis B virus (HBV) is the main causative agent of HCC in China. The present study aimed to investigate the potential mechanisms underlying HBV-related $\mathrm{HCC}$ and to identify core biomarkers by integrated bioinformatics analyses.

Methods: In the present study, HBV-related HCC GSE19665, GSE55092, GSE94660 and GSE121248 expression profiles were downloaded from the Gene Expression Omnibus database. These databases contain data for 299 samples, including 145 HBV-related HCC tissues and 154 non-cancerous tissues (from patients with chronic hepatitis B). The differentially expressed genes (DEGs) from each database were integrated and analyzed using the RobustRankAggreg (RRA) method and R software, and the integrated DEGs were identified. Subsequently, the gene ontology (GO) functional annotation and Kyoto Encyclopedia of Genes and Genomes (KEGG) pathway analysis were performed using the DAVID online tool, and the protein-protein interaction (PPI) network was constructed using STRING and visualized using Cytoscape software. Finally, hub genes were identified, and the cBioPortal online platform was used to analyze the association between the expression of hub genes and prognosis in HBV-related HCC. Results: First, 341 DEGs (117 upregulated and 224 downregulated) were identified from the four datasets. Next, GO analysis showed that the upregulated genes were mainly involved in cell cycle, mitotic spindle, and adenosine triphosphate binding. The majority of the downregulated genes were involved in oxidation reduction, extracellular region, and electron carrier activity. Signaling pathway analysis showed that the integrated DEGs shared common pathways in retinol metabolism, drug metabolism, tryptophan metabolism, caffeine metabolism, and metabolism of xenobiotics by cytochrome P450. The integrated DEG PPI network complex comprised 288 nodes, and two important modules with high degree were detected using the MCODE plug-in. The top ten hub genes identified from the PPI network were SHCBP1, FOXM1, KIF4A, ANLN, KIF15, KIF18A, FANCI, NEK2, ECT2, and RAD51AP1. Finally, survival analysis revealed that 
patients with HBV-related HCC showing altered ANLN and KIF18A expression profiles showed worse disease-free survival. Nonetheless, patients with FOXM1, NEK2, RAD51AP1, ANLN, and KIF18A alterations showed worse overall survival.

Conclusions: The present study identified key genes and pathways involved in HBV-related HCC, which improved our understanding of the mechanisms underlying the development and recurrence of HCC and identified candidate targets for the diagnosis and treatment of HBVrelated HCC.

\section{Introduction}

Hepatocellular carcinoma (HCC) is the sixth most commonly diagnosed type of malignant tumor and is the second leading cause of cancer-related deaths worldwide. It is estimated that there were about 841,000 new cases and 782,000 deaths caused by liver cancer worldwide in 2018 (Bray et al., 2018), with Chinese patients making up more than half of the global HCC burden (Jemal et al., 2011; Bray et al., 2018). The high incidence of HCC in parts of Asia is mainly due to the prevalence of hepatitis $\mathrm{B}$ virus and $\mathrm{C}$ virus infections, especially the hepatitis B virus (Bray et al., 2018). Accumulating evidence has shown that carcinogenesis and progression of $\mathrm{HCC}$ are closely related to overexpression of various oncogenes and inactivation of tumor suppressor genes.

The poor prognosis associated with HCC is attributed to the lack of effective diagnostic and therapeutic methods in the early stage of the disease. In recent years, gene targeting therapy has been increasingly used for the treatment of advanced HCC, and significant progress has been made. The most commonly reported genetic alterations in HCC include mutations in the TERT promoter, TP53, CTNB1, AXIN1, ARID1A, CDKN2A, ARID2, RPS6KA3, and CCND1 (Khemlina, Ikeda\&Kurzrock, 2017). Sorafenib targets multiple kinases and has been approved by the US Food and Drug Administration for the treatment of advanced HCC (Zhu et al., 2017). However, sorafenib has many shortcomings, such as low efficiency, high cost, and multiple side effects (Hu et al., 2013). Therefore, there is an urgent need to explore the relationship between the new gene function and the occurrence, development, and malignant characteristics of HCC, as well as to elucidate the precise molecular mechanisms underlying HCC, develop early screening methods, and discover novel and effective therapeutic strategies.

Recently, high - throughput technologies and gene chips have served as rapid methods for the identification of differentially expressed genes (DEGs) and functional pathways involved in the initiation and development of various diseases (Roh et al., 2010; Vogelstein et al., 2013). In these studies, a large number of tumor samples can be analyzed and thousands of genes can be identified; as a result, bioinformatics methods have become necessary for the analysis of gene expression profiles. However, obtaining reliable results from a single gene expression profile data is difficult, considering the potentially large number of differentially expressed genes, lack of stability and reproducibility, and high false-positive rates.

The RobustRankAggreg (RRA), which is based on a statistical model, is a biological analysis method for the integration and analysis of multiple gene lists (Kolde et al., 2012). RRA is a rank aggregation algorithm that assumes that all genes are arranged randomly in each dataset. 
A gene with a higher ranking in all datasets has a lower P-value and has a higher likelihood of being a DEG. Compared to other strategies used for the meta-analysis of datasets from multiple databases, the RRA method is more robust and easier to compute and facilitates better evaluation of the significance of the results. In addition, the RRA algorithm can handle the variable number of genes identified from different microarray platforms. More importantly, the RRA method does not strictly require the use of certain subset of problems or complete datasets to produce highly reliable results (Kolde et al., 2012). Therefore, the RRA algorithm is highly suitable for the integrated analysis of datasets from multiple databases.

In the present study, four GSE datasets GSE19665 (Deng et al., 2010), GSE55092 (Melis et al., 2014), GSE94660 (Yoo et al., 2017), and GSE121248 (Wang, Ooi\&Hui, 2007) were downloaded from GEO; these datasets comprise a total of 299 samples, including 145 hepatitis B virus (HBV)-related HCC tissues and 154 non-cancerous tissues (chronic hepatitis B patients). The chip probe IDs were converted to their corresponding gene symbols. Bioinformatics analysis using R software and RRA method was then performed to obtain the integrated differentially expressed genes (DEGs). The Gene Ontology (GO; http://www.geneontology.org) is a public bioinformatics resource that provides information about gene product function using ontologies to represent biological knowledge (Gaudet\&Dessimoz, 2017). KEGG (Kyoto Encyclopedia of Genes and Genomes) is a knowledgebase used for the systematic analysis of gene functions and for linking genomic information with higher-order functional information (Kanehisa\&Goto, 2000).Herein, enriched GO terms and KEGG pathways were identified using the online tool DAVID 6.7.Then, the protein-protein interaction (PPI) network of the DEGs was constructed, and the hub genes were identified. We constructed the network using the hub genes and their coexpressed genes and analyzed the biological processes associated with the hub genes. Finally, survival analysis was performed based on the hub DEGs by generating the Kaplan-Meier curves in the cBioPortal. Therefore, the hub DEGs and the associated enriched pathways identified in this study can serve as reliable molecular markers for HBV-related HCC.

\section{Material and methods \\ Microarray data}

Gene expression profiles of GSE19665, GSE55092, GSE94660 and GSE121248 were acquired from the National Center for Biotechnology Information (NCBI) Gene Expression Omnibus (GEO) database (https://www.ncbi.nlm.nih.gov/geo/).GSE19665, GSE55092 and GSE121248 dataset were based on the platforms of GPL570 [HG-U133_Plus_2] Affymetrix Human Genome U133 Plus 2.0 Array, while the platform of the GSE94660 dataset was GPL16791Illumina HiSeq 2500 (Homo sapiens). GSE19665, GSE55092, GSE94660, GSE121248 contain 5, 91, 21 and 37 cases of non-cancerous tissues from chronic hepatitis B patients, and 5, 49, 21 and 70 cases of HBV related HCC tissues respectively. The series matrix TXT files and platform TXT files of the four databases were downloaded separately, and the information is shown in Table 1. To obtain the international standard gene name, the process of the conversion of gene probe IDs in the matrix files to the gene symbols in the platform files was performed by using A Perl language command. Subsequently, the gene expression data, normalized by the normalization Between Arrays function, was subjected to $\log 2$ transformation 
123 in the limma R package (http://www.bioconductor.org/) (Ritchie et al., 2015). Mean values of

124

125

126

127

128

129

130

131

132

133

134

135

136

137

138

139

140

141

142

143

144

145

146

147

148

149

150

151

152

153

154

155

156

157

158

159

160

161

162

163

$\log _{2} \mathrm{FC}$ was used when multiple probe sets are used for one gene.

\section{Screening for DEGs}

The limma R package V3.5.2 in R software was used to identify DEGs in each dataset. The DEGs were screened out according to the cut-off criterion that adjusted P-value $<0.05$ and $\left|\log _{2} \mathrm{FC}\right|>\quad$ 1. The RobustRankAggreg (RRA) R package (https://cran.rstudio.com/bin/windows/contrib/3.5/RobustRankAggreg_1.1.zip)(Kolde et al., 2012) was used to integrated and analyzed the four gene lists which were sorted by $\operatorname{logFC}$ value. The lists of significantly upregulated and downregulated genes were exported and saved as Excel files respectively.

\section{GO and KEGG pathway enrichment analyses of DEGs}

The Database for Annotation, Visualization and Integrated Discovery (DAVID; http://david.ncifcrf.gov) (version 6.8) (Dennis et al., 2003; Huang et al., 2007), a common online program, integrates biological data and analysis tools to provide a comprehensive set of functional annotation information of large-scale lists of genes or proteins for users to grasp biological characteristics(Huang, Sherman\&Lempicki, 2009). In order to understand the selected DEGs better, GO and KEGG pathway enrichment analysis were executed by using DAVID online tool. $P<0.05$ was considered statistically significant.

\section{PPI network construction and module analysis}

As a public online tool, the Search Tool for the Retrieval of Interacting Genes (STRING) (https://string-db.org/) is designed to construct a critical assessment and intergration of PPI network, including direct (physical) and indirect (function) association (Szklarczyk et al., 2015). To know the interactional correlation of the DEGs, PPI network was established by STRING and then displayed using Cytoscape software (3.7.1) that is a public bioinformatics software platform (Shannon et al., 2003). Furthermore, the plug-in Molecular Complex Detection (MCODE) app in Cytoscape software (Bader\&Hogue, 2003) was also applied to select the significant modules of hub genes from the PPI network (degree cut-off $\geq 2$, node score cut-off $\geq 0.2, \mathrm{~K}$-core $\geq 2$, and max depth $=100$ ). Moreover, the KEGG and GO analyses for DEGs in modules were used to investigate their potential information by using DAVID.

\section{Hub genes selection and analysis}

The hub genes were selected with degrees $\geq 10$. The cBioPortal (http://www.cbioportal.org) online platform (Cerami et al., 2012; Gao et al., 2013) was used to analyze the network of the hub genes and their co-expression genes. The plug-in Biological Networks Gene Oncology tool (BiNGO) (version 3.0.3)(Maere, Heymans\&Kuiper, 2005) in Cytoscape software was used to construct and visualize the biological process analysis of hub genes. The UCSC Cancer Genomics Browser (http://genome-cancer.ucsc.edu) (Goldman et al., 2015) was applied to construct hierarchical clustering of hub genes. The overall survival and disease-free survival of hub genes were analyzed using the Kaplan-Meier curve in the HCC datasets (TCGA, Provisional) of the cBioPortal. Comparison of expression of these genes in multiple databases were analyzed using online database Oncomine (http://www.oncomine.com) (Rhodes et al., 2004).

\section{Results}

Peer] reviewing PDF | (2019:03:35556:2:1:NEW 19 Jun 2019) 


\section{Identification of DEGs in HCC}

Four HBV-related HCC gene expression profiles were downloaded from the NCBI GEO database. Afterwards, the gene expression data was normalized and DEGs were identified with the limma R package (adjusted $P<0.05$ and $\mid \log$ fold change $(\mathrm{FC}) \mid>1$ ), and the results are shown in Figure S1.We screened out 648, 1043, 1171, and 580 DEGs respectively (Table 2, Figure 1, Table S1). Through the integration and analysis of RRA, a total of integrated 341 DEGs were identified from the four datasets, including 117 upregulated genes and 224 downregulated genes (Table S2). The top 20 upregulated genes and the top 20 downregulated genes were charted on a heat map, as shown in Figure 2.

\section{Functional enrichment analyses of DEGs}

To further investigate the biological functions of the 314 DEGs, GO analysis was performed using online database DAVID 6.7. As shown in Figure 3A,B and Table 3, GO analysis can be divided into three functional groups: biological process group (BP), the cellular component group (CC), and the molecular function group (MF). The results of GO analysis exhibited that the integrated DEGs were particularly enriched in the BP, including cell cycle, $\mathrm{M}$ phase, cell cycle phase, mitosis and nuclear division for the upregulated DEGs and oxidation reduction, innate immune response, complement activation, response to wounding and activation of plasma proteins involved in acute inflammatory response for the downregulated DEGs. For the CC, the upregulated DEGs were mainly enriched in mitotic spindle, microtubule cytoskeleton, cytoskeletal part, cytoskeleton and spindle pole and the downregulated DEGs were enriched in extracellular region, extracellular region part, extracellular space, microsome, vesicular fraction. In the MF, the upregulated DEGs were principally enriched in ATP binding, adenyl ribonucleotide binding, adenyl nucleotide binding, purine nucleoside binding, nucleoside binding, and the downregulated DEGs were enriched in electron carrier activity, oxygen binding, iron ion binding, heme binding, tetrapyrrole binding.

\section{Signaling pathway enrichment analysis}

KEGG pathway enrichment analysis was performed using online database DAVID 6.7. As shown in Figure 3C and Table 4, the results revealed that the integrated DEGs were particularly enriched in retinol metabolism, drug metabolism, metabolism of xenobiotics by cytochrome P450, caffeine metabolism and tryptophan metabolism.

\section{Integration of PPI network and module analysis}

PPI network of the 341 DEGs was established by STRING. A total of 288 DEGs (99 upregulated genes and 189 downregulated genes) were filtered into the DEG PPI network complex, which contained 288 nodes and 2259 edges (Figure S2, Table S3,S4). Among the 288 nodes, 59 central node genes were selected as hub genes with the criteria of filtering degree $\geq 10$ (ie, each node has more than 10 connections/interactions). The top 10 hub genes were as follows: SHCBP1, FOXM1, KIF4A, ANLN, KIF15, KIF18A, FANCI, NEK2, ECT2 and RAD51AP1 (Table 5).

Furthermore, the two most significant modules (Figure 4A, 4B, Table S5, S6) of the PPI network were selected for KEGG pathway enrichment analysis. Results showed that the genes in module 1 were mainly enriched in cell cycle, oocyte meiosis, p53 signaling pathway, 
205

206

207

208

209

210

211

212

213

214

215

216

217

218

219

220

221

222

223

224

225

226

227

228

229

230

231

232

233

234

235

236

237

238

239

240

241

242

243

244

245

progesterone-mediated oocyte maturation, and the genes in module 2 were mainly enriched in complement and coagulation cascades, prion diseases and systemic lupus erythematosus (Table 6).

\section{Hub gene selection and analysis}

The biological process of the hub genes was analyzed and visualized using BiNGO in Cytoscape software and the result is shown in Figure 5A. The network of hub genes and their co-expression genes was constructed using cBioPortal online platform. As show in Figure 5B, the network contained 106 nodes, including 56 query genes and the 50 most frequently altered neighbor genes. Hierarchical cluster analysis showed that the high expression of hub genes was mainly in the region of HCC samples, whereas the low expression of hub genes was mainly in the region of non-HCC samples (Figure 4C). Subsequently, the prognostic information (overall survival and disease-free survival analyses) of the top 10 hub genes was available in the HCC datasets (TCGA, Provisional) of the cBioPortal online platform. HCC patients with ANLN and KIF18A alteration showed worse disease-free survival. Nonetheless, the patients with FOXM1, NEK2, RAD51AP1, ANLN, and KIF18A alteration showed worse overall survival (Figure 6).

Among these genes, ANLN and KIF18A showed the highest node degrees with 43 . Then, HCC patients with the two genes alteration showed worse overall survival and disease-free survival. Moreover, Oncomine analysis of cancer vs. normal tissue showed that ANLN and KIF18A were highly expressed in multiple HCC datasets (Figure 7). These findings indicate that they may play important roles in the carcinogenesis or progression of HBV-HCC.

\section{Discussion}

$\mathrm{HCC}$ is a common malignant tumor affecting the digestive system. The incidence of HCC in developing countries is considerably higher than that in developed countries. Chronic infection with $\mathrm{HBV}$ or hepatitis $\mathrm{C}$ virus $(\mathrm{HCV})$ is the primary etiological factor related to $\mathrm{HCC}$ in certain parts of Asia and sub-Saharan Africa(Bray et al., 2018). In western countries, the main risk factors include obesity, type 2 diabetes, heavy alcohol consumption, and smoking (El-Serag, 2011; Mittal\&El-Serag, 2013). Although significant progress has been achieved in the diagnosis and treatment of HCC in recent years, the prognosis of HCC remains poor (Okajima et al., 2017). Thus, there is an urgent need to understand the detailed molecular mechanisms underlying HCC for early detection, diagnosis, and treatment of the disease. Recently, the wide application of microarray and high-throughput technologies has provided an effective way to screen thousands of genes that are likely to be involved in the occurrence and development of HCC. These genes could serve as potential targets for the diagnosis and treatment of HCC.

In the present study, we analyzed four mRNA expression datasets and identified a total of 341 DEGs, comprising 117 upregulated genes and 224 downregulated genes, in HBV-related HCC samples. GO and KEGG enrichment analyses showed that the upregulated genes were associated with the cell cycle (ontology: BP), mitotic spindle (ontology: CC), and adenosine triphosphate binding, adenyl ribonucleotide binding, adenyl nucleotide binding, purine nucleoside binding, and nucleoside binding (ontology: MF). The majority of the downregulated genes were enriched in oxidation reduction (ontology: BP), extracellular region (ontology: CC), and electron carrier activity (ontology: MF). The above results suggested that these DEGs are 
246 involved in the proliferation and cell cycle of chronic hepatitis B-induced HCC cells. KEGG 247 pathway analysis revealed that the integrated DEGs were significantly enriched in retinol 248 metabolism, drug metabolism, metabolism of xenobiotics by cytochrome P450, caffeine

249

250

251

252

253

254

255

256

257

258

259

260

261

262

263

264

265

266

267

268

269

270

271

272

273

274

275

276

277

278

279

280

281

282

283

284

285

286 metabolism, and tryptophan metabolism. Previous studies have shown that dysregulation of the cell cycle and oxidation reduction play vital roles in the initiation or progression of tumors (Choi et al., 2001; Wang et al., 2017). Cytochrome P450 enzymes are involved in various types of cancer via several mechanisms, including the catalysis of the bioactivation of chemical procarcinogens, participation in the activation of cancer therapeutic drugs, as targets for cancer treatment, and as metabolic enzymes (Hrycay\&Bandiera, 2015).

After screening out two modules with the most significant interactions, ten genes with the highest degrees of interaction were subsequently identified by constructing the PPI network. Results of survival analysis showed that the patients with alterations in FOXM1, NEK2, RAD51AP1, ANLN, and KIF18A expression patterns showed worse prognosis.

ANLN, an actin-binding protein, has been reported to be dysregulated in diverse human tumors (Hall et al., 2005). ANLN is not only considered as a proliferative marker, but also a prognostic and therapeutic indicator. ANLN knockdown was demonstrated to inhibit cell growth and migration in breast cancer (Zhou et al., 2015). In addition, another study showed that high ANLN levels in the nuclear fraction are involved in the cell cycle and are correlated with poor prognosis (Magnusson et al., 2016). Upregulation of ANLN expression plays an important role in non-small cell lung cancers (NSCLC) by activating RHOA and participating in the phosphoinositide 3-kinase/AKT pathway (Suzuki et al., 2005). A previous study showed that ANLN expression in gastric cancer (GC) is a molecular predictor of intestinal and proliferative type gastric tumors (Pandi et al., 2014). Furthermore, ANLN was identified as a biomarker for the prognosis of bladder urothelial carcinoma (Zeng et al., 2017), colorectal cancer (Wang et al., 2016), and lung adenocarcinoma (Long et al., 2018).

ANLN mRNA levels were upregulated in human HCC tissues compared to non-tumor liver tissues. ANLN knockdown was demonstrated to slow down the growth rates of tumors, reduce the number of tumors, and prolong the survival of mice (Zhang et al., 2018). Consistent with the findings reported in previous studies (Lian et al., 2018), our results showed that higher ANLN expression levels are associated with worse clinical outcomes and a shorter survival times of patients with HCC, thereby highlighting the potential use of ANLN as a prognostic biomarker.

As a member of the kinesin-8 family, KIF18A plays crucial roles in regulating microtubule dynamics, chromosome congression, and cell division (Mayr et al., 2007). In fact, elevated KIF18A expression was observed in several human cancers. KIF18A overexpression in human breast cancer has been closely associated with tumor grade, metastasis, and poor survival (Zhang et al., 2010; Kasahara et al., 2016). Some researchers even advocate measurement of KIF18A levels in patients with estrogen receptor positive $(\mathrm{ER}+)$ breast cancer $(\mathrm{BC})$ prior to receiving endocrine therapy (Alfarsi et al., 2018). KIF18A expression levels were found to positively contribute to tumor stage, lymphatic invasion, lymph node metastasis venous invasion, and peritoneal dissemination in CRC (Nagahara et al., 2011). Proteomic analysis indicated that KIF18A is a promising biomarker for the early diagnosis of cholangiocarcinoma (CCA) 
287

288

289

290

291

292

293

294

295

296

297

298

299

300

301

302

303

304

305

306

307

308

309

310

311

312

313

314

315

316

317

318

319

320

321

322

323

324

325

326

(Rucksaken et al., 2012).

KIF18A expression levels were found to be markedly higher in liver cancer tissues compared to adjacent normal liver tissues (Liao et al., 2014). KIF18A has been suggested to promote proliferation, invasion, and metastasis of $\mathrm{HCC}$ cells by activating cell cycle signaling pathway and the Akt and MMP-7/MMP-9-related signaling pathways (Luo et al., 2018). KIF18A levels were found to be significantly related to clinicopathologic factors associated with alpha-

fetoprotein (AFP) concentrations $(\geqslant 200 \mathrm{ng} / \mathrm{mL})$, tumor size $(\geqslant 5 \mathrm{~cm})$, clinical tumor-nodemetastasis (TNM) stage, and portal vein tumor thrombus (PVTT). In survival analysis, TCGA, Provisional higher KIF18A expression had worse prognosis (shorter DFS and OS) (Liao et al., 2014). The above results indicated that KIF18A could serve as a novel biomarker for the diagnosis and treatment of HCC. Our findings are consistent with previous studies and demonstrated that previous experiments did not show whether these $\mathrm{HCC}$ were associated with HBV. Therefore, the role of KIF18A in HBV-related HCC types should be verified by further experiments.

As a member of the forkhead box (Fox) transcription factor family, FOXM1 is acts as an oncogene in many tumors, such as breast, cervix, and prostate cancers, and is known to play crucial roles in the prognosis and chemoresistance of tumors (Zhu et al., 2018). FOXM1 mRNA levels were upregulated in human HCC tissues and had positive relevance to the development, metastasis, recurrence, and worse clinical outcomes in HCC patients after orthotopic liver transplantation (Sun et al., 2011; Dai et al., 2015). SHCBP1, KIF4A, and ECT2 have been reported to mediate tumor initiation and progression of human HCC (Tao et al., 2013; Chen et al., 2015; Hou et al., 2017). NEK2 could serve as a useful predictor and potential therapeutic target in HCC (Fu et al., 2017). However, previous studies reported low NEK2 expression levels, which are inconsistent with our current findings. Other research groups reported that abnormal KIF15 levels were evidently associated with HCC progression and prognosis (Chen et al., 2017); however, these findings were not verified by cell or animal experiments. Similarly, these studies did not show that the occurrence of these HCCs is closely related to HBV.

FANCI and RAD51AP1 have been identified as new markers for HBV-related HCC, but have not been widely reported based on literature retrieval. Some findings provided new insights that RAD51AP1 is likely to mediate the molecular mechanisms underlying HCV-induced pathogenesis (Nguyen et al., 2018). However, further studies are required to verify the exact roles of these two genes.

In accordance with our findings, previous studies have also identified DEGs that participate in HBV-related liver cancer (Zhou et al., 2017). For example, Zhou et al. analyzed the gene expression profiles of GSE14520 and HCC samples from the Zhongshan Hospital affiliated with Fudan University, which comprised 63 paired HCC and non-tumor samples. All patients of these two cohorts were infected with hepatitis B virus. A total of 965 DEGs (389 upregulated genes and 576 downregulated genes) that were differentially regulated by at least two-fold with statistical significance. HSP90AB1, RPL8, NPM1, and MCM3 were selected as the hub genes from the PPI network. Nevertheless, the study by Zhou et al. comprised relatively fewer samples 
327

328

329

330

331

332

333

334

335

336

337

338

339

340

341

342

343

344

345

346

(66 primary HCC tumors and paired adjacent non-tumor tissues), and the main purpose of the study was to identify copy number variation (CNV)-driven DEGs. The DEGs that are common from four datasets were visualized using a Venn diagram (Chen et al., 2019). As a result, the number of DEGs obtained using this method was relatively small (84 upregulated and 46 downregulated). In the end, the following top ten hub genes were obtained: TOP2A, RFC4, CCNB1, CDC20, CDKN3, BUB1B, CCNB2, TPX2, PEN1, and MAD2L1. Compared to the previous two studies, we analyzed four GEO datasets comprising 299 samples, and 341 DEGs (117 upregulated and 224 downregulated) were identified. In addition, data analysis was conducted using the RobustRankAggreg (RRA) method, which is highly suitable for the analysis of datasets from multiple databases. Therefore, the hub genes identified in the present study are more reliable and comprehensive.

\section{Conclusion}

In summary, by conducting an integrated bioinformatics analysis using multiple datasets, we identified DEGs and the association pathways involved in HBV-induced HCCs. In addition, we identified several key candidate genes and biological pathways that can provide a deeper and more comprehensive understanding of the occurrence and development of HCC and its association with HBV. Our findings provided valuable insights for the identification of novel biomarkers for the diagnosis and treatment of HCC.

\section{References:}

Alfarsi LH, Elansari R, Toss MS, Diez-Rodriguez M, Nolan CC, Ellis IO, Rakha EA, Green AR. 2018. Kinesin family member-18A (KIF18A) is a predictive biomarker of poor benefit from endocrine therapy in early ER+ breast cancer. Breast Cancer Res Treat. DOI 10.1007/s10549-018-4978-5

Bader GD, Hogue CW. 2003. An automated method for finding molecular complexes in large protein interaction networks. BMC Bioinformatics 4:2. DOI

Bray F, Ferlay J, Soerjomataram I, Siegel RL, Torre LA, Jemal A. 2018. Global cancer statistics 2018: GLOBOCAN estimates of incidence and mortality worldwide for 36 cancers in 185 countries. CA Cancer J Clin 68(6):394-424. DOI 10.3322/caac.21492

Cerami E, Gao J, Dogrusoz U, Gross BE, Sumer SO, Aksoy BA, Jacobsen A, Byrne CJ, Heuer ML, Larsson E, Antipin Y, Reva B, Goldberg AP, Sander C, Schultz N. 2012. The cBio cancer genomics portal: an open platform for exploring multidimensional cancer genomics data. Cancer Discov 2(5):401-4. DOI 10.1158/2159-8290.CD-12-0095

Chen J, Li S, Zhou S, Cao S, Lou Y, Shen H, Yin J, Li G. 2017. Kinesin superfamily protein expression and its association with progression and prognosis in hepatocellular carcinoma. J Cancer Res Ther 13(4):651-659. DOI 10.4103/jcrt.JCRT_491_17

Chen J, Xia H, Zhang X, Karthik S, Pratap SV, Ooi LL, Hong W, Hui KM. 2015. ECT2 regulates the Rho/ERK signalling axis to promote early recurrence in human hepatocellular carcinoma. $J$ Hepatol 62(6):1287-95. DOI 10.1016/j.jhep.2015.01.014

Chen Z, Chen J, Huang X, Wu Y, Huang K, Xu W, Xie L, Zhang X, Liu H. 2019. Identification of 
Potential Key Genes for Hepatitis B Virus-Associated Hepatocellular Carcinoma by Bioinformatics Analysis. $J$ Comput Biol. DOI 10.1089/cmb.2018.0244

Chen Z, Chen J, Huang X, Wu Y, Huang K, Xu W, Xie L, Zhang X, Liu H. 2019. Identification of Potential Key Genes for Hepatitis B Virus-Associated Hepatocellular Carcinoma by Bioinformatics Analysis. $J$ Comput Biol. DOI 10.1089/cmb.2018.0244

Choi YL, Park SH, Jang JJ, Park CK. 2001. Expression of the G1-S modulators in hepatitis B virus-related hepatocellular carcinoma and dysplastic nodule: association of cyclin D1 and p53 proteins with the progression of hepatocellular carcinoma. $J$ Korean Med Sci 16(4):424-32. DOI 10.3346/jkms.2001.16.4.424

Dai J, Yang L, Wang J, Xiao Y, Ruan Q. 2015. Prognostic Value of FOXM1 in Patients with Malignant Solid Tumor: A Meta-Analysis and System Review. Dis Markers 2015:352478. DOI 10.1155/2015/352478

Deng YB, Nagae G, Midorikawa Y, Yagi K, Tsutsumi S, Yamamoto S, Hasegawa K, Kokudo N, Aburatani H, Kaneda A. 2010. Identification of genes preferentially methylated in hepatitis $\mathrm{C}$ virus-related hepatocellular carcinoma. Cancer Sci 101(6):1501-10. DOI 10.1111/j.1349-7006.2010.01549.x

Dennis GJ, Sherman BT, Hosack DA, Yang J, Gao W, Lane HC, Lempicki RA. 2003. DAVID: Database for Annotation, Visualization, and Integrated Discovery. Genome Biol 4(5):P3. DOI

El-Serag HB. 2011. Hepatocellular carcinoma. $N$ Engl $J$ Med 365(12):1118-27. DOI 10.1056/NEJMra1001683

Fu L, Liu S, Wang H, Ma Y, Li L, He X, Mou X, Tong X, Hu Z, Ru G. 2017. Low expression of NEK2 is associated with hepatocellular carcinoma progression and poor prognosis. Cancer Biomark 20(1):101-106. DOI 10.3233/CBM-170586

Gao J, Aksoy BA, Dogrusoz U, Dresdner G, Gross B, Sumer SO, Sun Y, Jacobsen A, Sinha R, Larsson E, Cerami E, Sander C, Schultz N. 2013. Integrative analysis of complex cancer genomics and clinical profiles using the cBioPortal. Sci Signal 6(269):pl1. DOI 10.1126/scisignal.2004088

Gaudet P, Dessimoz C. 2017. Gene Ontology: Pitfalls, Biases, and Remedies. Methods Mol Biol 1446:189205. DOI 10.1007/978-1-4939-3743-1_14

Goldman M, Craft B, Swatloski T, Cline M, Morozova O, Diekhans M, Haussler D, Zhu J. 2015. The UCSC Cancer Genomics Browser: update 2015. Nucleic Acids Res 43(Database issue):D812-7. DOI 10.1093/nar/gku1073

Hall PA, Todd CB, Hyland PL, McDade SS, Grabsch H, Dattani M, Hillan KJ, Russell SE. 2005. The septin-binding protein anillin is overexpressed in diverse human tumors. Clin Cancer Res 11(19 Pt 1):6780-6. DOI 10.1158/1078-0432.CCR-05-0997

Hou G, Dong C, Dong Z, Liu G, Xu H, Chen L, Liu L, Wang H, Zhou W. 2017. Upregulate KIF4A Enhances Proliferation, Invasion of Hepatocellular Carcinoma and Indicates poor prognosis Across Human Cancer Types. Sci Rep 7(1):4148. DOI 10.1038/s41598-017-04176-9

Hrycay EG, Bandiera SM. 2015. Involvement of Cytochrome P450 in Reactive Oxygen Species Formation and Cancer. Adv Pharmacol 74:35-84. DOI 10.1016/bs.apha.2015.03.003

Hu Y, Wang S, Wu X, Zhang J, Chen R, Chen M, Wang Y. 2013. Chinese herbal medicine-derived compounds for cancer therapy: a focus on hepatocellular carcinoma. $J$ Ethnopharmacol 149(3):601-12. DOI 10.1016/j.jep.2013.07.030

Huang DW, Sherman BT, Lempicki RA. 2009. Systematic and integrative analysis of large gene lists using DAVID bioinformatics resources. Nat Protoc 4(1):44-57. DOI 10.1038/nprot.2008.211

Peer) reviewing PDF | (2019:03:35556:2:1:NEW 19 Jun 2019) 
Huang DW, Sherman BT, Tan Q, Collins JR, Alvord WG, Roayaei J, Stephens R, Baseler MW, Lane HC, Lempicki RA. 2007. The DAVID Gene Functional Classification Tool: a novel biological module-centric algorithm to functionally analyze large gene lists. Genome Biol 8(9):R183. DOI 10.1186/gb-2007-8-9-r183

Jemal A, Bray F, Center MM, Ferlay J, Ward E, Forman D. 2011. Global cancer statistics. CA Cancer J Clin 61(2):69-90. DOI 10.3322/caac.20107

Kanehisa M, Goto S. 2000. KEGG: kyoto encyclopedia of genes and genomes. Nucleic Acids Res 28(1):27-30. DOI

Kasahara M, Nagahara M, Nakagawa T, Ishikawa T, Sato T, Uetake H, Sugihara K. 2016. Clinicopathological relevance of kinesin family member 18A expression in invasive breast cancer. Oncol Lett 12(3):1909-1914. DOI 10.3892/ol.2016.4823

Khemlina G, Ikeda S, Kurzrock R. 2017. The biology of Hepatocellular carcinoma: implications for genomic and immune therapies. Mol Cancer 16(1):149. DOI 10.1186/s12943-017-0712-x

Kolde R, Laur S, Adler P, Vilo J. 2012. Robust rank aggregation for gene list integration and meta-analysis. Bioinformatics 28(4):573-80. DOI 10.1093/bioinformatics/btr709

Kolde R, Laur S, Adler P, Vilo J. 2012. Robust rank aggregation for gene list integration and meta-analysis. Bioinformatics 28(4):573-80. DOI 10.1093/bioinformatics/btr709

Lian YF, Huang YL, Wang JL, Deng MH, Xia TL, Zeng MS, Chen MS, Wang HB, Huang YH. 2018. Anillin is required for tumor growth and regulated by miR-15a/miR-16-1 in HBV-related hepatocellular carcinoma. Aging (Albany NY) 10(8):1884-1901. DOI 10.18632/aging.101510

Liao W, Huang G, Liao Y, Yang J, Chen Q, Xiao S, Jin J, He S, Wang C. 2014. High KIF18A expression correlates with unfavorable prognosis in primary hepatocellular carcinoma. Oncotarget 5(21):10271-9. DOI 10.18632/oncotarget.2082

Long X, Zhou W, Wang Y, Liu S. 2018. Prognostic significance of ANLN in lung adenocarcinoma. Oncol Lett 16(2):1835-1840. DOI 10.3892/ol.2018.8858

Luo W, Liao M, Liao Y, Chen X, Huang C, Fan J, Liao W. 2018. The role of kinesin KIF18A in the invasion and metastasis of hepatocellular carcinoma. World J Surg Oncol 16(1):36. DOI 10.1186/s12957-018-13425

Maere S, Heymans K, Kuiper M. 2005. BiNGO: a Cytoscape plugin to assess overrepresentation of gene ontology categories in biological networks. Bioinformatics 21(16):3448-9. DOI 10.1093/bioinformatics/bti551

Magnusson K, Gremel G, Ryden L, Ponten V, Uhlen M, Dimberg A, Jirstrom K, Ponten F. 2016. ANLN is a prognostic biomarker independent of $\mathrm{Ki}-67$ and essential for cell cycle progression in primary breast cancer. BMC Cancer 16(1):904. DOI 10.1186/s12885-016-2923-8

Mayr MI, Hummer S, Bormann J, Gruner T, Adio S, Woehlke G, Mayer TU. 2007. The human kinesin Kif18A is a motile microtubule depolymerase essential for chromosome congression. Curr Biol 17(6):488-98. DOI 10.1016/j.cub.2007.02.036

Melis M, Diaz G, Kleiner DE, Zamboni F, Kabat J, Lai J, Mogavero G, Tice A, Engle RE, Becker S, Brown CR, Hanson JC, Rodriguez-Canales J, Emmert-Buck M, Govindarajan S, Kew M, Farci P. 2014. Viral expression and molecular profiling in liver tissue versus microdissected hepatocytes in hepatitis B virus-associated hepatocellular carcinoma. $J$ Transl Med 12:230. DOI 10.1186/s12967-014-0230-1

Mittal S, El-Serag HB. 2013. Epidemiology of hepatocellular carcinoma: consider the population. J Clin Gastroenterol 47 Suppl:S2-6. DOI 10.1097/MCG.0b013e3182872f29 
Nagahara M, Nishida N, Iwatsuki M, Ishimaru S, Mimori K, Tanaka F, Nakagawa T, Sato T, Sugihara K, Hoon DS, Mori M. 2011. Kinesin 18A expression: clinical relevance to colorectal cancer progression. Int $J$ Cancer 129(11):2543-52. DOI 10.1002/ijc.25916

Nguyen T, Park EM, Lim YS, Hwang SB. 2018. Nonstructural Protein 5A Impairs DNA Damage Repair: Implications for Hepatitis C Virus-Mediated Hepatocarcinogenesis. J Virol 92(11). DOI 10.1128/JVI.00178-18

Okajima W, Komatsu S, Ichikawa D, Miyamae M, Ohashi T, Imamura T, Kiuchi J, Nishibeppu K, Arita T, Konishi H, Shiozaki A, Morimura R, Ikoma H, Okamoto K, Otsuji E. 2017. Liquid biopsy in patients with hepatocellular carcinoma: Circulating tumor cells and cell-free nucleic acids. World J Gastroenterol 23(31):56505668. DOI 10.3748/wjg.v23.i31.5650

Pandi NS, Manimuthu M, Harunipriya P, Murugesan M, Asha GV, Rajendran S. 2014. In silico analysis of expression pattern of a Wnt/beta-catenin responsive gene ANLN in gastric cancer. Gene 545(1):23-9. DOI 10.1016/j.gene.2014.05.013

Rhodes DR, Yu J, Shanker K, Deshpande N, Varambally R, Ghosh D, Barrette T, Pandey A, Chinnaiyan AM. 2004. ONCOMINE: a cancer microarray database and integrated data-mining platform. Neoplasia 6(1):1-6. DOI

Ritchie ME, Phipson B, Wu D, Hu Y, Law CW, Shi W, Smyth GK. 2015. limma powers differential expression analyses for RNA-sequencing and microarray studies. Nucleic Acids Res 43(7):e47. DOI 10.1093/nar/gkv007

Roh SW, Abell GC, Kim KH, Nam YD, Bae JW. 2010. Comparing microarrays and next-generation sequencing technologies for microbial ecology research. Trends Biotechnol 28(6):291-9. DOI 10.1016/j.tibtech.2010.03.001

Rucksaken R, Khoontawad J, Roytrakul S, Pinlaor P, Hiraku Y, Wongkham C, Pairojkul C, Boonmars T, Pinlaor S. 2012. Proteomic analysis to identify plasma orosomucoid 2 and kinesin $18 \mathrm{~A}$ as potential biomarkers of cholangiocarcinoma. Cancer Biomark 12(2):81-95. DOI 10.3233/CBM-130296

Shannon P, Markiel A, Ozier O, Baliga NS, Wang JT, Ramage D, Amin N, Schwikowski B, Ideker T. 2003. Cytoscape: a software environment for integrated models of biomolecular interaction networks. Genome Res 13(11):2498-504. DOI 10.1101/gr.1239303

Sun H, Teng M, Liu J, Jin D, Wu J, Yan D, Fan J, Qin X, Tang H, Peng Z. 2011. FOXM1 expression predicts the prognosis in hepatocellular carcinoma patients after orthotopic liver transplantation combined with the Milan criteria. Cancer Lett 306(2):214-22. DOI 10.1016/j.canlet.2011.03.009

Suzuki C, Daigo Y, Ishikawa N, Kato T, Hayama S, Ito T, Tsuchiya E, Nakamura Y. 2005. ANLN plays a critical role in human lung carcinogenesis through the activation of RHOA and by involvement in the phosphoinositide 3-kinase/AKT pathway. Cancer Res 65(24):11314-25. DOI 10.1158/0008-5472.CAN-05-1507

Szklarczyk D, Franceschini A, Wyder S, Forslund K, Heller D, Huerta-Cepas J, Simonovic M, Roth A, Santos A, Tsafou KP, Kuhn M, Bork P, Jensen LJ, von Mering C. 2015. STRING v10: protein-protein interaction networks, integrated over the tree of life. Nucleic Acids Res 43(Database issue):D447-52. DOI 10.1093/nar/gku1003

Tao HC, Wang HX, Dai M, Gu CY, Wang Q, Han ZG, Cai B. 2013. Targeting SHCBP1 inhibits cell proliferation in human hepatocellular carcinoma cells. Asian Pac J Cancer Prev 14(10):5645-50. DOI

Vogelstein B, Papadopoulos N, Velculescu VE, Zhou S, Diaz LJ, Kinzler KW. 2013. Cancer genome landscapes. Science 339(6127):1546-58. DOI 10.1126/science.1235122

Peer) reviewing PDF | (2019:03:35556:2:1:NEW 19 Jun 2019) 
490

491

492

493

494

495

496

497

498

499

500

501

502

503

504

505

506

507

508

509

510

511

512

513

514

515

516

517

518

519

520

521

Wang B, Fu J, Yu T, Xu A, Qin W, Yang Z, Chen Y, Wang H. 2017. Contradictory effects of mitochondriaand non-mitochondria-targeted antioxidants on hepatocarcinogenesis by altering DNA repair in mice. Hepatology. DOI 10.1002/hep.29518

Wang G, Shen W, Cui L, Chen W, Hu X, Fu J. 2016. Overexpression of Anillin (ANLN) is correlated with colorectal cancer progression and poor prognosis. Cancer Biomark 16(3):459-65. DOI 10.3233/CBM-160585

Wang SM, Ooi LL, Hui KM. 2007. Identification and validation of a novel gene signature associated with the recurrence of human hepatocellular carcinoma. Clin Cancer Res 13(21):6275-83. DOI 10.1158/1078-0432.CCR-062236

Yoo S, Wang W, Wang Q, Fiel MI, Lee E, Hiotis SP, Zhu J. 2017. A pilot systematic genomic comparison of recurrence risks of hepatitis B virus-associated hepatocellular carcinoma with low- and high-degree liver fibrosis. BMC Med 15(1):214. DOI 10.1186/s12916-017-0973-7

Zeng S, Yu X, Ma C, Song R, Zhang Z, Zi X, Chen X, Wang Y, Yu Y, Zhao J, Wei R, Sun Y, Xu C. 2017. Transcriptome sequencing identifies ANLN as a promising prognostic biomarker in bladder urothelial carcinoma. Sci Rep 7(1):3151. DOI 10.1038/s41598-017-02990-9

Zhang C, Zhu C, Chen H, Li L, Guo L, Jiang W, Lu SH. 2010. Kif18A is involved in human breast carcinogenesis. Carcinogenesis 31(9):1676-84. DOI 10.1093/carcin/bgq134

Zhang S, Nguyen LH, Zhou K, Tu HC, Sehgal A, Nassour I, Li L, Gopal P, Goodman J, Singal AG, Yopp A, Zhang Y, Siegwart DJ, Zhu H. 2018. Knockdown of Anillin Actin Binding Protein Blocks Cytokinesis in Hepatocytes and Reduces Liver Tumor Development in Mice Without Affecting Regeneration. Gastroenterology 154(5):1421-1434. DOI 10.1053/j.gastro.2017.12.013

Zhou C, Zhang W, Chen W, Yin Y, Atyah M, Liu S, Guo L, Shi Y, Ye Q, Dong Q, Ren N. 2017. Integrated Analysis of Copy Number Variations and Gene Expression Profiling in Hepatocellular carcinoma. Sci Rep 7(1):10570. DOI 10.1038/s41598-017-11029-y

Zhou W, Wang Z, Shen N, Pi W, Jiang W, Huang J, Hu Y, Li X, Sun L. 2015. Knockdown of ANLN by lentivirus inhibits cell growth and migration in human breast cancer. Mol Cell Biochem 398(1-2):11-9. DOI 10.1007/s11010-014-2200-6

Zhu Q, Sun Y, Zhou Q, He Q, Qian H. 2018. Identification of key genes and pathways by bioinformatics analysis with TCGA RNA sequencing data in hepatocellular carcinoma. Mol Clin Oncol 9(6):597-606. DOI 10.3892/mco.2018.1728

Zhu YJ, Zheng B, Wang HY, Chen L. 2017. New knowledge of the mechanisms of sorafenib resistance in liver cancer. Acta Pharmacol Sin 38(5):614-622. DOI 10.1038/aps.2017.5

Peer] reviewing PDF | (2019:03:35556:2:1:NEW 19 Jun 2019) 
Figure 1

Figure 1 Differential expression genes between the two groups of samples in each dataset.

(A) GSE19665, (B) GSE55092, (C) GSE94660, (D) GSE121248. The red dots represent the upregulated genes based on an adjusted $\mathrm{P}<0.05$ and log fold change $>1$; the green dots represent the downregulated genes based on an adjusted $P<0.05$ and log fold change $<1$; the black spots represent genes with no significant difference in expression. 
A
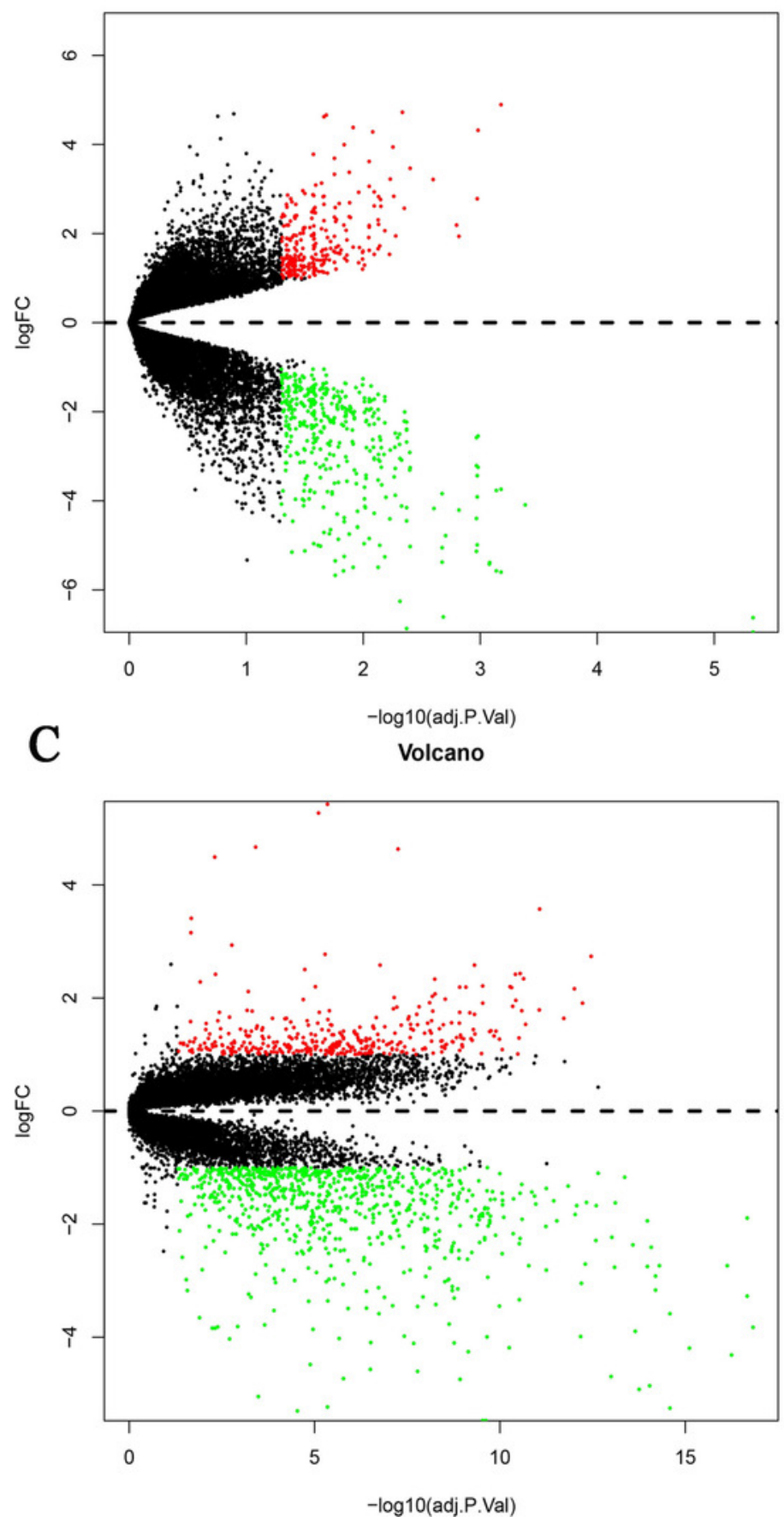

B Volcano
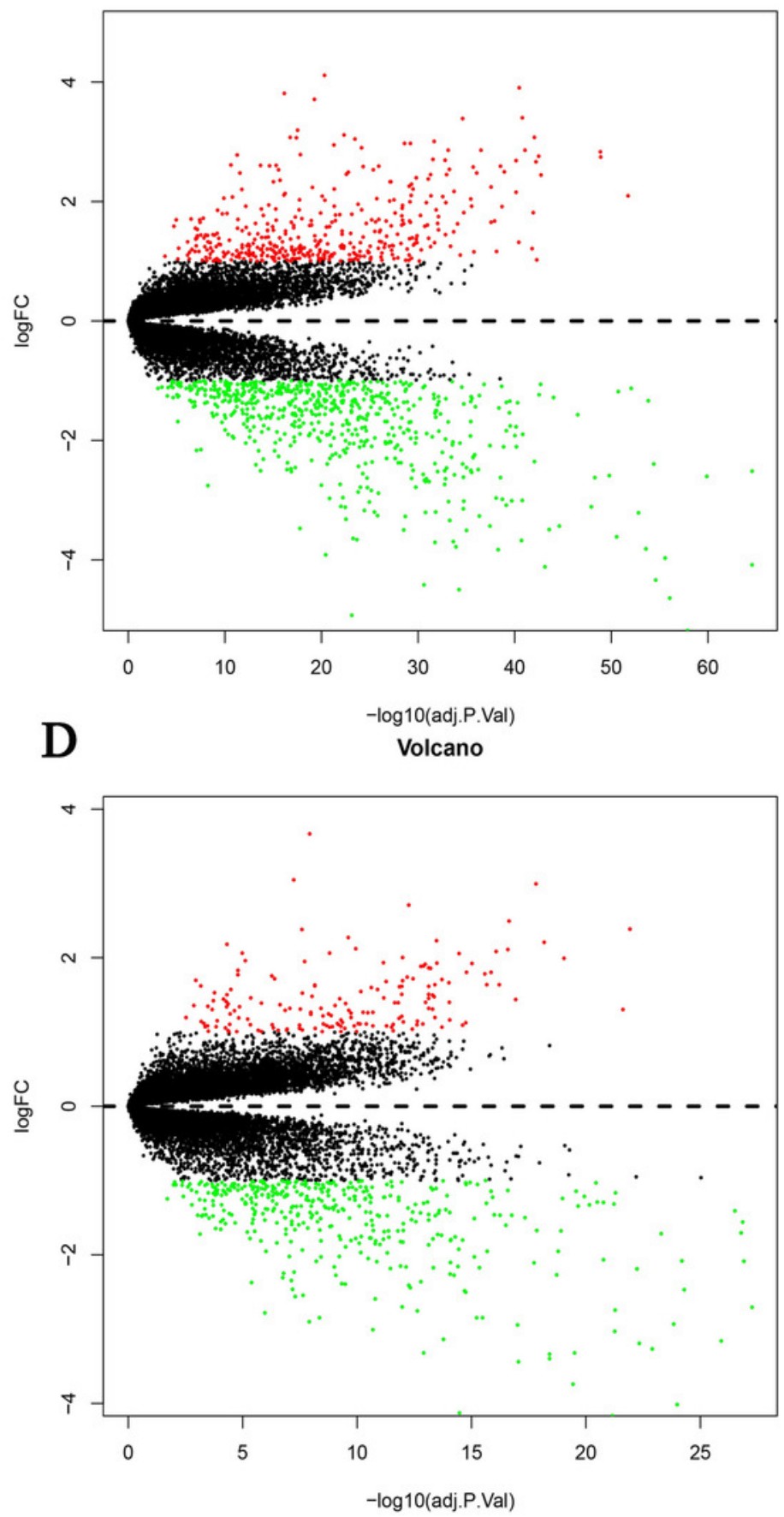
Figure 2

Figure 2 Log FC Heatmap of the top 20 DEGs (upregulated genes and downregulated genes) expression in all datasets.

The abscissa represent the GEO IDs, the ordinate represents the gene name, the red represents $\log \mathrm{FC}>0$, the white represents $\log \mathrm{FC}=0$, the green represents $\log \mathrm{FC}<0$ and the value in the box represents the log FC value. 


\begin{tabular}{|c|c|c|c|c|}
\hline 3.80 & 2.59 & & 2.71 & GPC3 \\
\hline 2.94 & 2.50 & 1.77 & 1.91 & FAM83D \\
\hline 2.90 & 2.45 & 3.57 & 2.21 & TOP2A \\
\hline 2.88 & 3.05 & 2.42 & 1.83 & LCN2 \\
\hline 2.92 & 2.16 & 1.98 & 1.92 & ASPM \\
\hline 2.84 & 2.83 & 2.01 & 2.39 & CAP2 \\
\hline 2.65 & 2.54 & 1.53 & 2.09 & PRC1 \\
\hline 2.62 & 2.68 & 2.42 & 1.89 & CCNB1 \\
\hline 2.77 & 1.98 & 1.64 & 1.31 & IGF2BP3 \\
\hline \multirow[t]{3}{*}{1.29} & 4.12 & 2.94 & 3.67 & SPINK1 \\
\hline & 3.07 & 0.53 & 2.12 & RBM24 \\
\hline & 2.40 & 1.36 & 1.93 & NUF2 \\
\hline \multirow[t]{2}{*}{2.36} & 2.10 & 1.74 & 1.39 & KIF11 \\
\hline & 3.39 & 0.85 & 2.06 & ZIC2 \\
\hline 2.48 & 2.25 & 1.30 & 1.93 & GINS1 \\
\hline 3.77 & 3.08 & -0.05 & 2.18 & MAGEA6 \\
\hline 2.66 & 2.58 & 1.27 & 2.23 & PBK \\
\hline 2.23 & 3.40 & 1.84 & 2.00 & ROBO1 \\
\hline 2.20 & 1.98 & 1.51 & 1.72 & KIF4A \\
\hline 2.19 & 1.52 & 1.33 & 1.78 & NUSAP1 \\
\hline-6.95 & -5.19 & -5.47 & -4.13 & HAMP \\
\hline-5.60 & -3.97 & -4.86 & -3.32 & FCN3 \\
\hline-5.33 & -3.69 & -4.10 & -2.85 & MT1M \\
\hline-6.87 & -3.49 & -4.70 & -3.03 & CRHBP \\
\hline-5.00 & -4.93 & -4.73 & -2.78 & $\mathrm{Cg}$ \\
\hline-6.26 & -3.62 & -3.83 & -3.19 & CLEC4G \\
\hline-4.71 & -3.50 & -3.99 & -3.74 & CNDP1 \\
\hline-5.49 & -3.11 & -4.92 & -2.94 & TTC36 \\
\hline-4.86 & -3.02 & -4.26 & -2.70 & APOF \\
\hline-5.50 & -3.22 & -3.77 & -2.17 & SLC25A47 \\
\hline-5.57 & -4.08 & -3.28 & -3.16 & CLEC1B \\
\hline-4.46 & -2.79 & -3.78 & -2.23 & CYP2A6 \\
\hline-4.15 & -3.15 & -5.48 & -3.40 & CYP1A2 \\
\hline-5.25 & -4.42 & -3.10 & -2.54 & SLC22A1 \\
\hline-4.07 & -2.62 & -3.42 & -2.10 & CD5L \\
\hline-5.38 & -2.61 & -3.31 & -2.71 & CXCL14 \\
\hline-4.23 & -2.52 & -4.32 & -2.08 & FCN2 \\
\hline-4.04 & -2.86 & -2.87 & -2.31 & GYS2 \\
\hline-4.99 & -4.34 & -2.77 & -3.27 & OIT3 \\
\hline-3.83 & -2.36 & -4.61 & -2.34 & THRSP \\
\hline 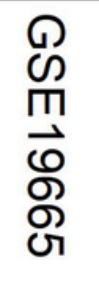 & $\begin{array}{l}\text { O } \\
\text { O } \\
\text { Un } \\
\text { G } \\
\text { N } \\
\text { N }\end{array}$ & 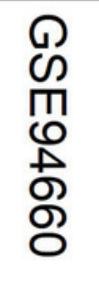 & 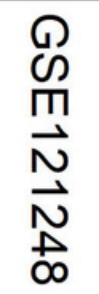 & \\
\hline
\end{tabular}


Figure 3

Figure 3 (A) GO analysis of upregulated DEGs. (B) GO analysis of downregulated DEGs. (C) KEGG pathway of DEGs.

A

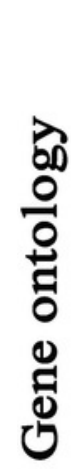

రิ
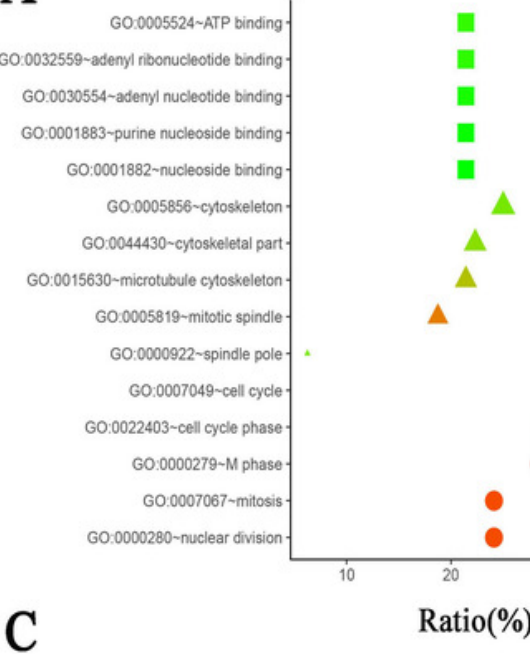

$$
\begin{aligned}
& \text { hsa00830:Retinol metabolism } \\
& \text { hsa00982:Drug metabolism }
\end{aligned}
$$

hsa00980:Metabolism of xenobiotics by cytochrome P450 hsa04110:Cell cycle hsa04610:Complement and coagulation cascades hsa00380:Tryptophan metabolism hsa00983:Drug metabolism hsa00590:Arachidonic acid metabolism hsa04115:p53 signaling pathway hsa04114:Oocyte meiosis hsa00591:Linoleic acid metabolism hsa00350:Tyrosine metabolism hsa05322:Systemic lupus erythematosus hsa00232:Caffeine metabolism hsa05020:Prion diseases hsa00140:Steroid hormone biosynthesis hsa00010: Glycolysis / Gluconeogenesis hsa00260: Glycine, serine and threonine metabolism -

\footnotetext{
hsa00071:Fatty acid metabolism
hsa00360:Phenylalanine metabolism

hsa00071:Fatty acid metabolism
hsa00360:Phenylalanine metabolism
}

B
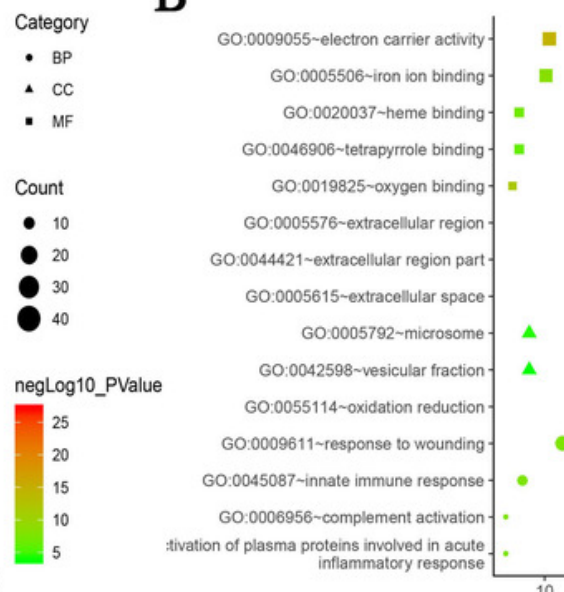

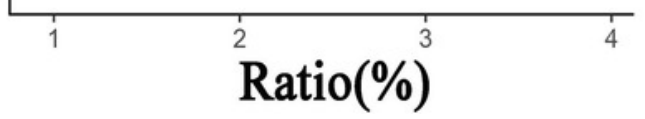

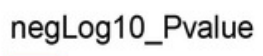

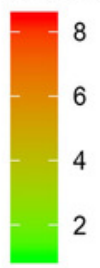

Count

- 5.0

7.5

10.0

12.5 


\section{Figure 4}

Figure 4 PPI network of module 1 (A), module 2 (B) and hierarchical clustering of hub genes was constructed using UCSC (C).

( $A$ and $B$ )Circles represent genes, lines represent interactions between gene-encoded proteins and line colors represent evidence of interactions between proteins. (C)The samples under the pink bar are normal samples and the samples under the blue bar are HCC samples. Upregulation of genes is marked in red; downregulation of genes is marked in blue 
A

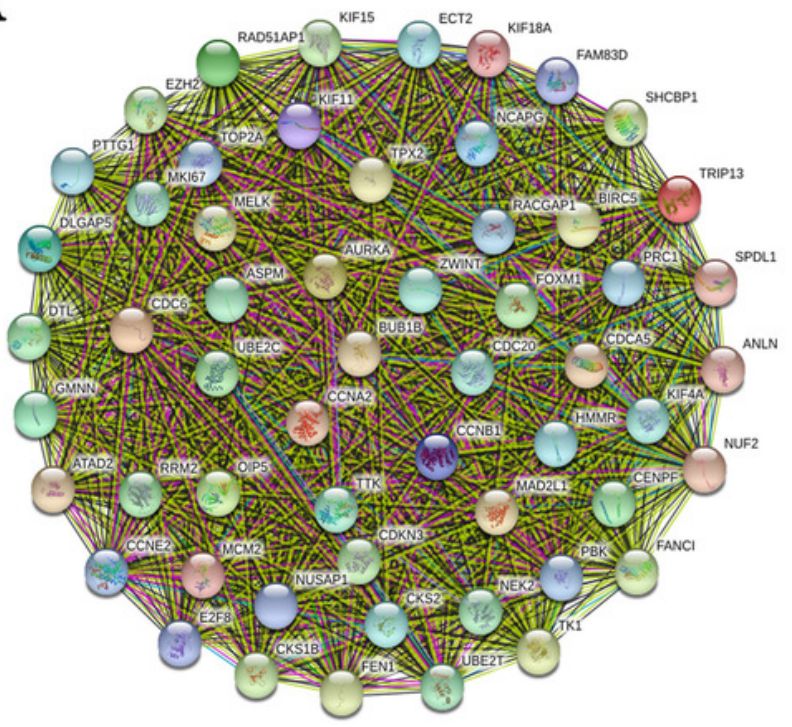

B

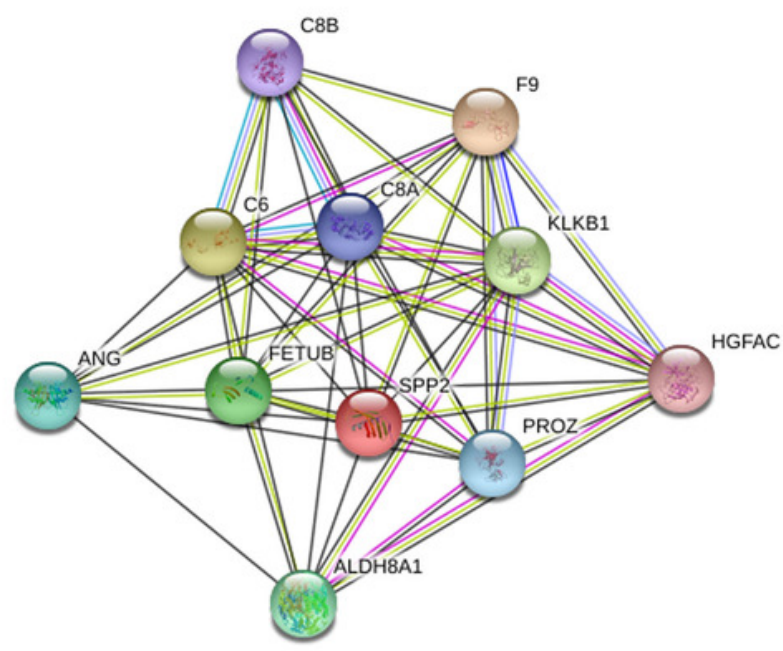

Sample type Gene expression Grade

C
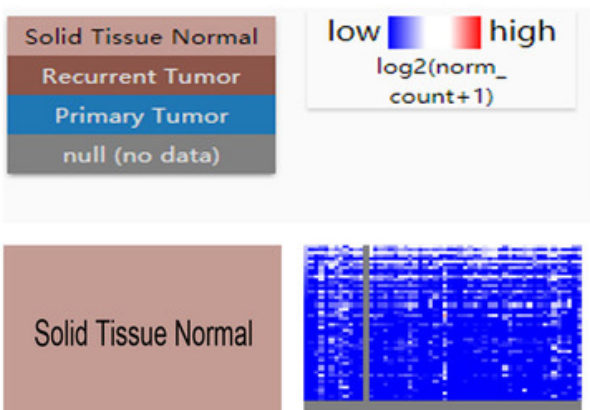

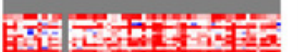

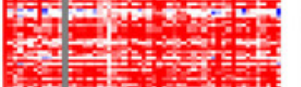

the

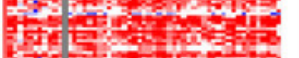

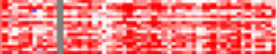

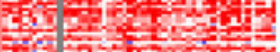

$x+50$ hom

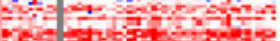

nestivin

is

的

证

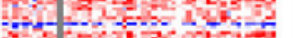

14 $-\frac{2}{2}$

Primary Tumor

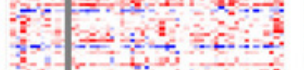

12 -1

iv $7+4$

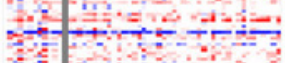

18

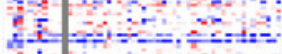

$+\therefore-1,-3$

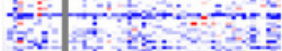

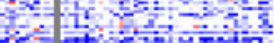

Le. bicinito

$402+3 x^{2}$

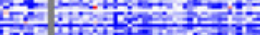

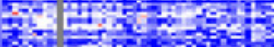

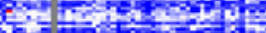

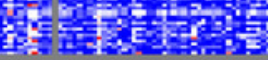

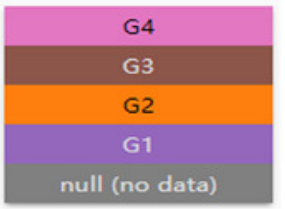

null (no data)

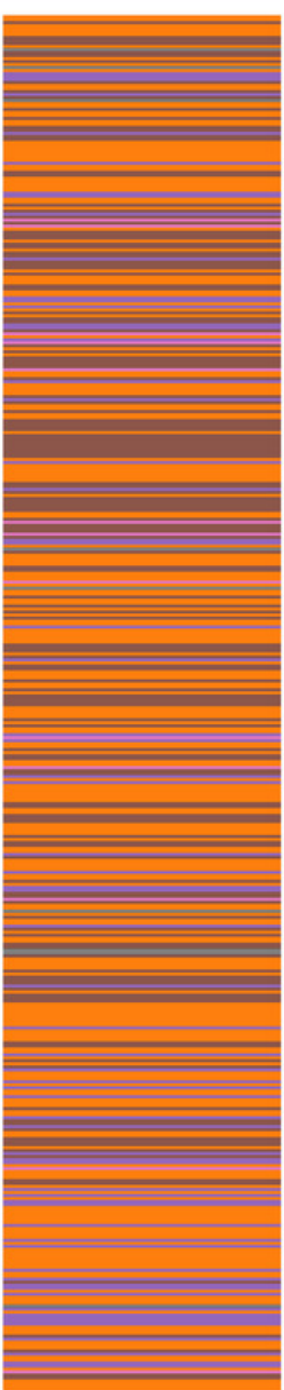




\section{Figure 5}

Figure 5 The biological process analysis of the hub genes. (A)The biological process analysis of hub genes was constructed using BiNGO.

(A)The biological process analysis of hub genes was constructed using BiNGO. The color depth of nodes refers to the corrected P-value of ontologies. The size of nodes refers to the numbers of genes that are involved in the ontologies. $\mathrm{P}<0.05$ was considered statistically significant. (B) Hub genes and their co-expression genes were analyzed using cBioPortal. Nodes with bold black outline represent hub genes. Nodes with thin black outline represent the co-expression genes. 


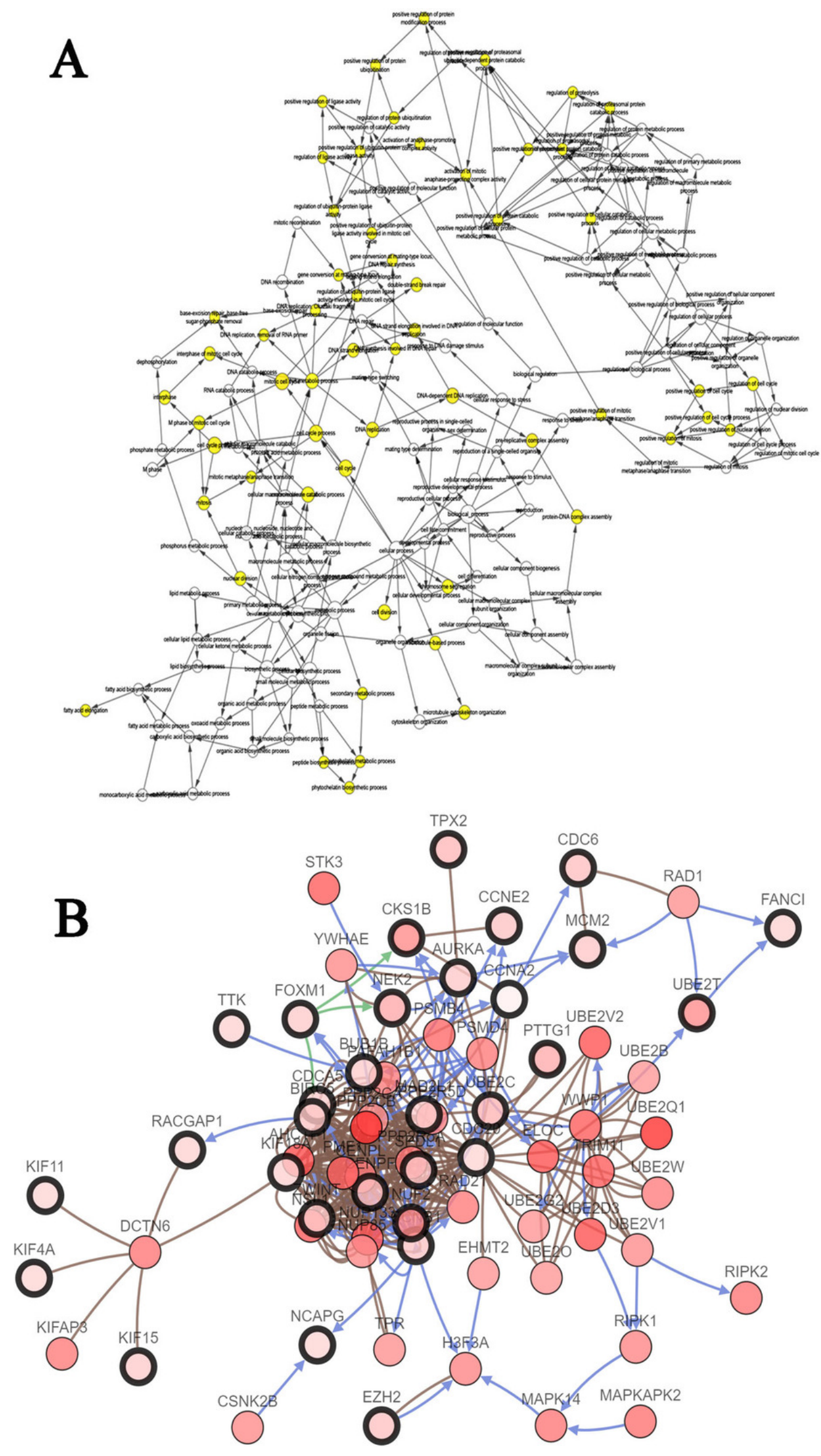

PeerJ reviewing PDF | (2019:03:35556:2:1:NEW 19 Jun 2019) 
Figure 6

Figure $6(A, B)$ Disease-free survival analyses and (C-G) overall survival of hub genes were performed using CBioPortal online platform.

$\mathrm{P}<0.05$ was considered statistically significant. 
A
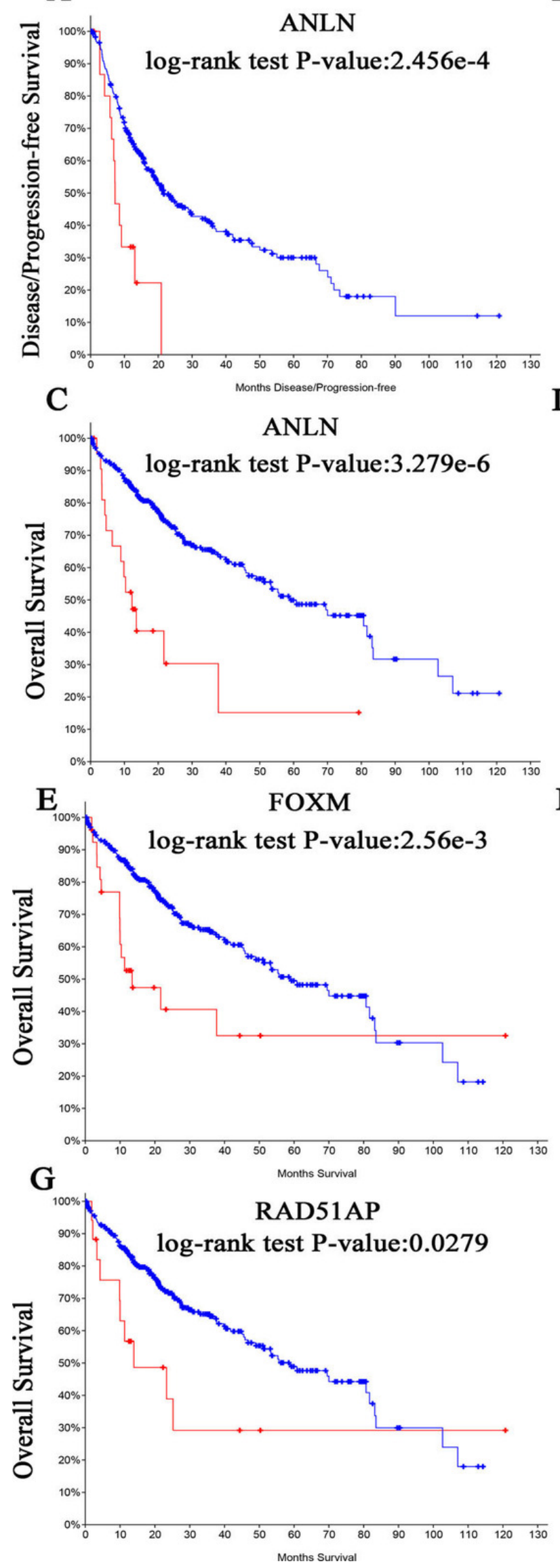

B
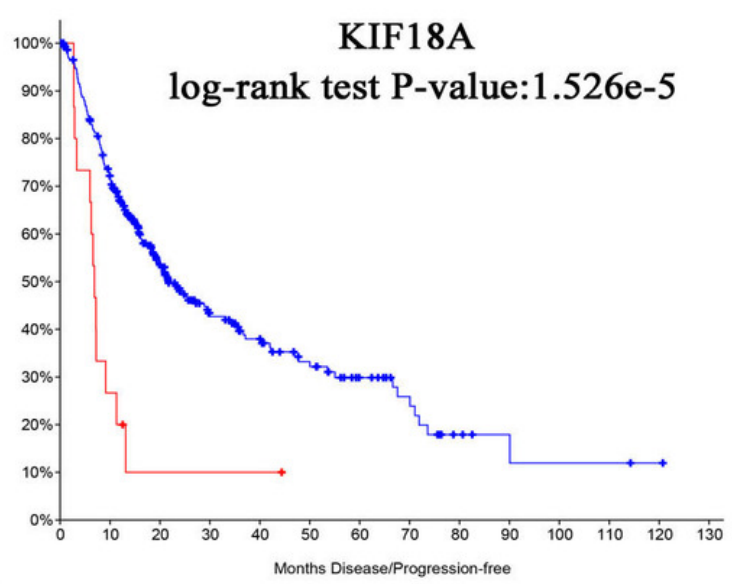

D

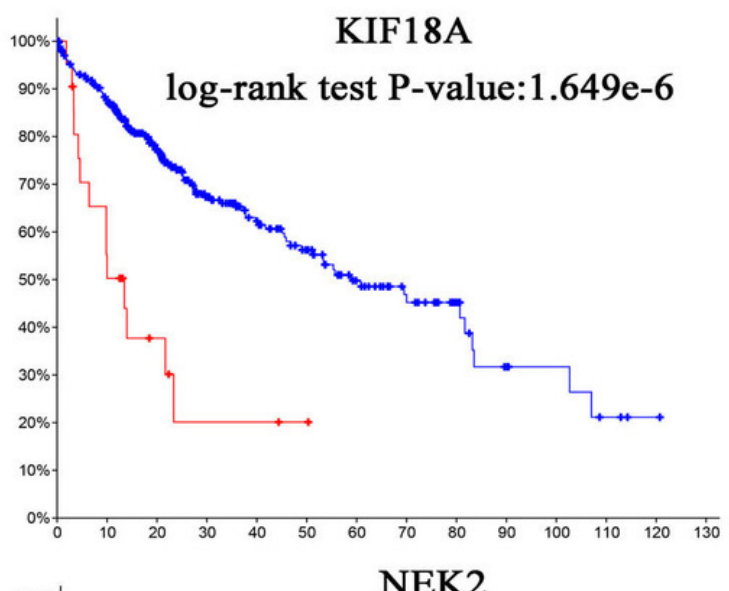

F

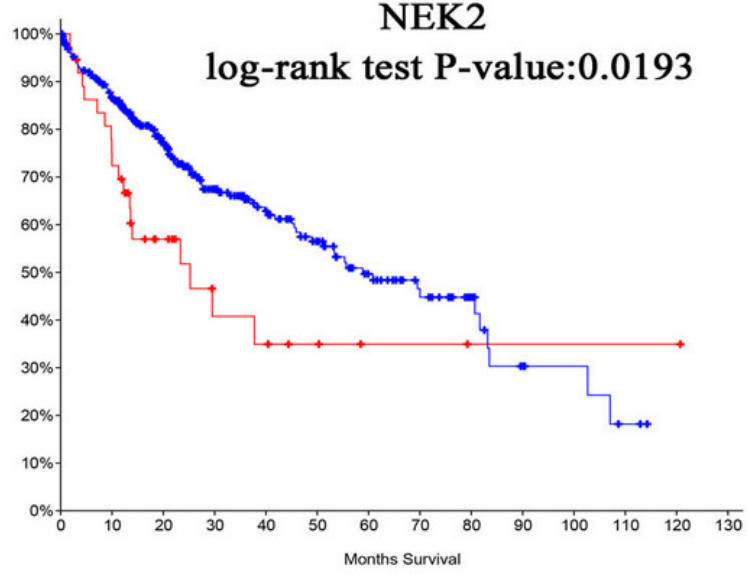


Figure 7

Figure 7 Heat maps of ANLN (A) and KIF18A (B) gene expression in multiple clinical hepatocellular carcinoma samples vs. normal tissues using Oncomine analysis.

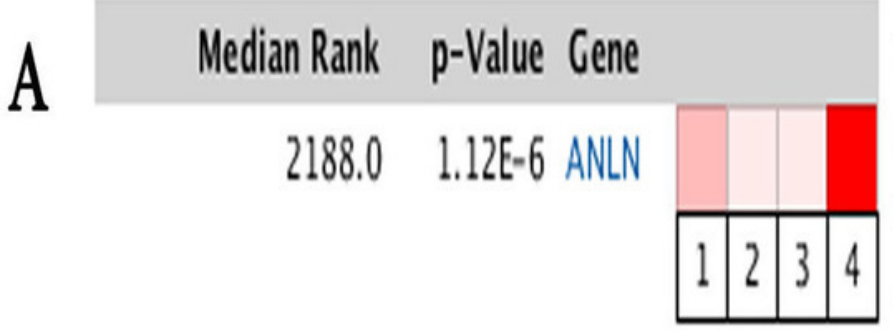

1P-value=7.82E-10 fold change=1.048 Guichard Liver, Nat Genet, 2012

2P-value $=0.024$ fold change $=1.023$ Guichard Liver 2, Nat Genet, 2012

3P-value=2.24E-6 fold change=1.074 TCGA Liver, No Associated Paper, 2012

4P-value=7.66E-11 fold change=7.344 Wurmbach Liver, Hepatology, 2007

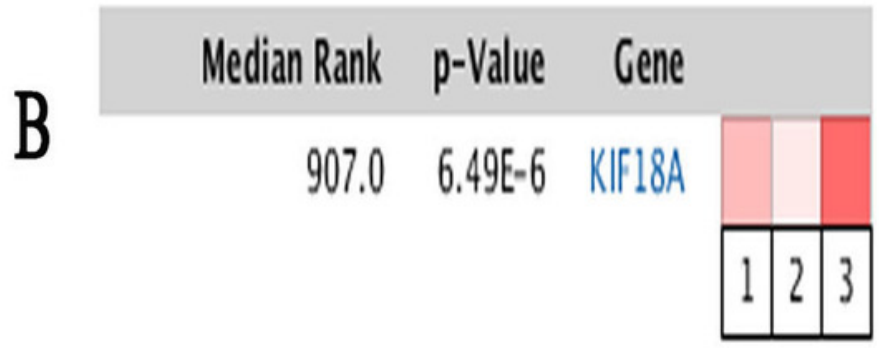

1P-value=6.49E-6 fold change=1.261 Roessler Liver, Cancer Res, 2010 2P-value $=1.58 \mathrm{E}-29$ fold change $=1.265$ Roessler Liver 2 , Cancer Res, 2010 3P-value $=3.4 \mathrm{E}-6$ fold change $=1.970$ Wurmbach Liver, Hepatology, 2007

151025251051

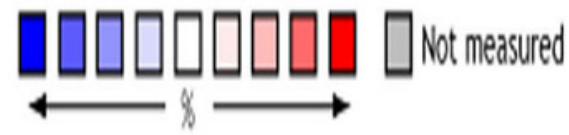


Table $\mathbf{1}$ (on next page)

Table 1: Details of the GEO HBV-related HCC data. 


\begin{tabular}{llllll}
\multicolumn{1}{c}{1} & Platform & Normal & Tumor & Reference \\
\hline GSE & Sample & GPL570 & 5 & 5 & Deng YB et al(2010) \\
GSE55092 & $\begin{array}{l}\text { carcinoma(HBV) } \\
\text { hepatocellular } \\
\text { carcinoma(HBV) } \\
\text { hepatocellular } \\
\text { carcinoma(HBV) } \\
\text { hepatocellular } \\
\text { carcinoma(HBV) }\end{array}$ & GPL570 & 91 & 49 & Melis M et al(2014) \\
GSE121248 & GPL570 & 37 & 70 & Wang SM et al(2007) \\
\hline
\end{tabular}

2 Note: GEO, gene expression omnibus. 
Table 2 (on next page)

Table 2: Information of DEGs screened from each dataset. 
1

\begin{tabular}{|c|c|c|c|c|}
\hline GEO & Sample & $\begin{array}{l}\text { Number of } \\
\text { DEGs }\end{array}$ & $\begin{array}{c}\text { Number of } \\
\text { upregulated } \\
\text { genes }\end{array}$ & $\begin{array}{c}\text { Number of } \\
\text { downregulated } \\
\text { genes }\end{array}$ \\
\hline GSE19665 & $\begin{array}{c}\text { hepatocellular } \\
\text { carcinoma(HBV) }\end{array}$ & 648 & 257 & 391 \\
\hline GSE55092 & $\begin{array}{c}\text { hepatocellular } \\
\text { carcinoma(HBV) }\end{array}$ & 1034 & 409 & 634 \\
\hline GSE94660 & $\begin{array}{c}\text { hepatocellular } \\
\text { carcinoma(HBV) }\end{array}$ & 1171 & 360 & 811 \\
\hline GSE121248 & $\begin{array}{c}\text { hepatocellular } \\
\text { carcinoma(HBV) }\end{array}$ & 580 & 167 & 413 \\
\hline
\end{tabular}

2 Note: GEO, gene expression omnibus.

3 


\section{Table 3 (on next page)}

Table 3: Top 15 GO enrichment terms of differentially expressed genes associated with hepatitis B-related hepatocellular carcinoma. 
1

\begin{tabular}{|c|c|c|c|c|c|}
\hline Expression & Category & Term & Count & $\%$ & PValue \\
\hline \multirow{15}{*}{ upregulated } & $\mathrm{BP}$ & GO:0007049 cell cycle & 43 & 38.39286 & $7.63 \mathrm{E}-28$ \\
\hline & $\mathrm{BP}$ & GO:0000279 M phase & 32 & 28.57143 & $2.48 \mathrm{E}-27$ \\
\hline & BP & GO:0022403 cell cycle phase & 33 & 29.46429 & $1.59 \mathrm{E}-25$ \\
\hline & $\mathrm{BP}$ & GO:0007067 mitosis & 27 & 24.10714 & 2.03E-25 \\
\hline & $\mathrm{BP}$ & GO:0000280 nuclear division & 27 & 24.10714 & 2.03E-25 \\
\hline & $\mathrm{CC}$ & GO:0005819 mitotic spindle & 21 & 18.75 & 1.43E-21 \\
\hline & $\mathrm{CC}$ & GO:0015630 microtubule cytoskeleton & 24 & 21.42857 & $2.84 \mathrm{E}-13$ \\
\hline & $\mathrm{CC}$ & GO:0044430 cytoskeletal part & 25 & 22.32143 & 3.19E-09 \\
\hline & $\mathrm{CC}$ & GO:0005856 cytoskeleton & 28 & 25 & $5.91 \mathrm{E}-08$ \\
\hline & $\mathrm{CC}$ & GO:0000922 spindle pole & 7 & 6.25 & $6.75 \mathrm{E}-08$ \\
\hline & MF & GO:0005524 ATP binding & 24 & 21.42857 & $3.45 \mathrm{E}-05$ \\
\hline & MF & $\begin{array}{c}\text { GO:0032559 adenyl ribonucleotide } \\
\text { binding }\end{array}$ & 24 & 21.42857 & 4.27E-05 \\
\hline & MF & GO:0030554 adenyl nucleotide binding & 24 & 21.42857 & $9.64 \mathrm{E}-05$ \\
\hline & MF & GO:0001883 purine nucleoside binding & 24 & 21.42857 & $1.22 \mathrm{E}-04$ \\
\hline & MF & GO:0001882 nucleoside binding & 24 & 21.42857 & $1.35 \mathrm{E}-04$ \\
\hline \multirow{15}{*}{ downregulated } & $\mathrm{BP}$ & GO:0055114 oxidation reduction & 36 & 16.66667 & $7.03 \mathrm{E}-14$ \\
\hline & $\mathrm{BP}$ & GO:0045087 innate immune response & 15 & 6.944444 & $1.67 \mathrm{E}-09$ \\
\hline & $\mathrm{BP}$ & GO:0006956 complement activation & 10 & 4.62963 & $1.78 \mathrm{E}-09$ \\
\hline & $\mathrm{BP}$ & GO:0009611 response to wounding & 27 & 12.5 & $1.84 \mathrm{E}-09$ \\
\hline & $\mathrm{BP}$ & $\begin{array}{l}\text { GO:0002541 activation of plasma proteins } \\
\text { involved in acute inflammatory response }\end{array}$ & 10 & 4.62963 & 2.23E-09 \\
\hline & $\mathrm{CC}$ & GO:0005576 extracellular region & 83 & 38.42593 & $2.05 \mathrm{E}-21$ \\
\hline & $\mathrm{CC}$ & GO:0044421 extracellular region part & 50 & 23.14815 & $9.40 \mathrm{E}-16$ \\
\hline & $\mathrm{CC}$ & GO:0005615 extracellular space & 41 & 18.98148 & $8.87 \mathrm{E}-15$ \\
\hline & $\mathrm{CC}$ & GO:0005792 microsome & 17 & 7.87037 & $2.50 \mathrm{E}-07$ \\
\hline & $\mathrm{CC}$ & GO:0042598 vesicular fraction & 17 & 7.87037 & $3.70 \mathrm{E}-07$ \\
\hline & MF & GO:0009055 electron carrier activity & 23 & 10.64815 & $1.17 \mathrm{E}-13$ \\
\hline & MF & GO:0019825 oxygen binding & 12 & 5.555556 & $5.61 \mathrm{E}-12$ \\
\hline & MF & GO:0005506 iron ion binding & 22 & 10.18519 & $5.88 \mathrm{E}-10$ \\
\hline & MF & GO:0020037 heme binding & 14 & 6.481481 & $6.05 \mathrm{E}-09$ \\
\hline & MF & GO:0046906 tetrapyrrole binding & 14 & 6.481481 & $1.33 \mathrm{E}-08$ \\
\hline
\end{tabular}

2 Notes: BP, biological process.CC, cellular component. MF, molecular function

3 GO: gene ontology 


\section{Table 4(on next page)}

Table 4: KEGG pathway analysis of differentially expressed genes associated with hepatitis B-related hepatocellular carcinoma. 
1

\section{Term}

\begin{tabular}{cc}
\hline Term & Co \\
hsa00830:Retinol metabolism & 1
\end{tabular}

hsa00982:Drug metabolism

hsa00980:Metabolism of

xenobiotics by cytochrome

P450

hsa00232:Caffeine metabolism

hsa00380:Tryptophan

metabolism

hsa00591:Linoleic acid

metabolism

hsa00983:Drug metabolism

hsa04610:Complement and

coagulation cascades

hsa00590:Arachidonic acid

metabolism

hsa04110:Cell cycle

hsa00350:Tyrosine metabolism

hsa04115:p53 signaling

pathway

hsa05020:Prion diseases

hsa00140:Steroid hormone

biosynthesis

hsa00260:Glycine, serine and

threonine metabolism

hsa04114:Oocyte meiosis

hsa00010:Glycolysis /

Gluconeogenesis

hsa00071:Fatty acid metabolism

hsa05322:Systemic lupus erythematosus

\section{Count}

13

3.963415

3.73E-09

$12 \quad 3.658537 \quad 2.06 \mathrm{E}-07$

$11 \quad 3.353659 \quad 1.39 \mathrm{E}-06$

$5 \quad 1.52439 \quad 1.11 \mathrm{E}-05$

$7 \quad 2.134146 \quad 3.62 \mathrm{E}-04$

$6 \quad 1.829268 \quad 4.98 \mathrm{E}-04$

$7 \quad 2.134146 \quad 5.42 \mathrm{E}-04$

$\begin{array}{lll}8 & 2.439024 & 0.001332\end{array}$

$\begin{array}{lll}7 & 2.134146 & 0.002231\end{array}$

$10 \quad 3.04878 \quad 0.003227$

$\begin{array}{lll}6 & 1.829268 & 0.004034\end{array}$

$\begin{array}{lll}7 & 2.134146 & 0.005932\end{array}$

$\begin{array}{lll}5 & 1.52439 & 0.009832\end{array}$

$\begin{array}{lll}5 & 1.52439 & 0.024978\end{array}$

$\begin{array}{lll}4 & 1.219512 & 0.038725\end{array}$

$\begin{array}{lll}7 & 2.134146 & 0.05109\end{array}$

$\begin{array}{lll}5 & 1.52439 & 0.057701\end{array}$

$4 \quad 1.219512 \quad 0.072811$

$6 \quad 1.829268 \quad 0.093168$

\section{Genes}

CYP3A4, CYP2B6, CYP2C9, CYP2C18, CYP2C8,

CYP26A1, ADH1A, CYP1A2, CYP4A11, ADH4, CYP2A6, CYP2A7, RDH16

CYP3A4, CYP2C18, CYP2C9, CYP2B6, ADH4, CYP2C8, GSTZ1, CYP2A6, ADH1A, CYP2A7, CYP1A2, ALDH3A1

AKR1C3, CYP3A4, CYP2C18, CYP2C9, CYP2B6, ADH4, CYP2C8, GSTZ1, ADH1A, CYP1A2, ALDH3A1

XDH, NAT2, CYP2A6, CYP2A7, CYP1A2

AADAT, TDO2, ACMSD, IDO2, KMO, CYP1A2,

INMT

CYP3A4, CYP2C18, CYP2C9, AKR1B10, CYP2C8, CYP1A2

CYP3A4, XDH, NAT2, CDA, CYP2A6, CYP2A7,

TK1

C8A, C8B, C7, C9, C6, KLKB1, F9, PLG

AKR1C3, CYP4A11, CYP2C18, CYP2C9, CYP2B6, CYP2C8, CYP4F2

CCNE2, CCNB1, CDC6, MAD2L1, BUB1B, TTK, CDC20, MCM2, PTTG1, CCNA2

ADH4, GSTZ1, ADH1A, TAT, ALDH3A1, HPD

STEAP3, CCNE2, CCNB1, RRM2, IGF1, THBS1, IGFBP3

C8A, C8B, C7, C9, C6

AKR1C3, CYP3A4, HSD17B2, SRD5A2, AKR1D1

SDS, AGXT2, GNMT, GLDC

CCNE2, CCNB1, MAD2L1, IGF1, CDC20, AURKA, PTTG1

ADH4, ALDOB, FBP1, ADH1A, ALDH3A1

CYP4A11, ADH4, ADH1A, ACSL4

C8A, C8B, C7, C9, C6, HIST1H4H 
hsa00360:Phenylalanine metabolism 


\section{Table 5 (on next page)}

Table 5: The top 10 most degree values hub genes between HBV-related HCC and normal samples.

UP indicated that the gene was identifed as up-regulated in HCC; Down indicated that the gene was reported as down-regulated. UN suggested the gene has not been reported in current HCC associated studies. 
1

\begin{tabular}{ccccc}
\hline Gene symbol & Gene description & logFC & Degree & Up/down \\
\hline SHCBP1 & SHC binding and spindle associated 1 & 1.211943105 & 43 & Up \\
FOXM1 & forkhead box M1 & 1.832558689 & 43 & Up \\
KIF4A & kinesin family member 4A & 1.850277648 & 43 & Up \\
ANLN & anillin actin binding protein & 1.982343654 & 43 & Up \\
KIF15 & kinesin family member 15 & 1.247277869 & 43 & UN \\
KIF18A & kinesin family member 18A & 1.26437819 & 43 & Up \\
FANCI & FA complementation group I & 1.269987824 & 43 & UN \\
NEK2 & NIMA related kinase 2 & 1.652396787 & 43 & Down \\
ECT2 & epithelial cell transforming 2 & 1.418475417 & 43 & Up \\
RAD51AP1 & RAD51 associated protein 1 & 1.585151756 & 42 & UN \\
\hline
\end{tabular}


Table 6(on next page)

Table 6: KEGG enrichment of genes in the top 2 modules. 
1

\begin{tabular}{|c|c|c|c|c|c|}
\hline Module & Term & Count & $\%$ & PValue & Genes \\
\hline \multirow{4}{*}{ Modul1 } & hsa04110:Cell cycle & 10 & 18.18182 & $5.06 \mathrm{E}-11$ & $\begin{array}{l}\text { CCNE2, CCNB1, CDC6, } \\
\text { MAD2L1, BUB1B, TTK, CDC20, } \\
\text { MCM2, PTTG1, CCNA2 }\end{array}$ \\
\hline & hsa04114:Oocyte meiosis & 6 & 10.90909 & $2.18 \mathrm{E}-05$ & $\begin{array}{l}\text { CCNE2, CCNB1, MAD2L1, } \\
\text { CDC20, AURKA, PTTG1 }\end{array}$ \\
\hline & hsa04115:p53 signaling pathway & 3 & 5.454545 & 0.021056 & CCNE2, CCNB1, RRM2 \\
\hline & $\begin{array}{l}\text { hsa04914:Progesterone-mediated } \\
\text { oocyte maturation }\end{array}$ & 3 & 5.454545 & 0.032616 & CCNB1, MAD2L1, CCNA2 \\
\hline \multirow{3}{*}{ Modul2 } & $\begin{array}{l}\text { hsa04610:Complement and } \\
\text { coagulation cascades }\end{array}$ & 5 & 45.45455 & $3.11 \mathrm{E}-08$ & C8A, C8B, C6, KLKB1, F9 \\
\hline & hsa05020:Prion diseases & 3 & 27.27273 & 2.74E-04 & C8A, C8B, C6 \\
\hline & $\begin{array}{l}\text { hsa05322:Systemic lupus } \\
\text { erythematosus }\end{array}$ & 3 & 27.27273 & 0.002195 & $\mathrm{C} 8 \mathrm{~A}, \mathrm{C} 8 \mathrm{~B}, \mathrm{C} 6$ \\
\hline
\end{tabular}

2

3 